\title{
To the question of the uniqueness of the reduction potential in the inverse problem of the Borg-Levinson
}

\author{
Smirnova L.V. \\ Nosov Magnitogorsk State Technical University, \\ the city of Magnitogorsk, Chelyabinsk oblast, Russia \\ email: smirnova20@bk.ru
}

Torshina O.A.

Nosov Magnitogorsk State Technical University, the city of Magnitogorsk, Chelyabinsk oblast, Russia e-mail: olganica@mail.ru

This paper reviews an inverse problem of spectral analysis of the Dirichlet's boundary value problem for Laplace operator with potential in a bounded domain $\Omega$ in $R^{2}$ or $R^{3}$.

Key words - inverse problem, Laplace operator, potential, recovery of the potential, sequences, Dirichlet's boundary value problem.

Under inverse problems of spectral analysis to understand the problem of reconstructing an operator from its predetermined spectral characteristics. In this study, hesitant recovery uniqueness of the operator from the spectral data, specified for different boundary conditions. Many inverse problems are not the only solution. Thus, for example, Borg showed that Sturm - Liouville generally not uniquely defined by one spectrum. Therefore, one of the most important is the problem of the uniqueness of the potential recovery in the solution which raises the question of the identification of additional conditions to ensure the uniqueness of the inverse problem solution.

For the first time the inverse problem for the Sturm Liouville was set VA Ambartsumian, and in its simplest formulation, it zaklyualas to identify the operator, knowing its spectrum. Further advances in the theory of inverse problems has been achieved as a result of the application to the study of inverse problems of the so-called conversion operators. This method was developed in detail in the works of VA Marchenko, MG Crane, IM Gelfand, BM Levitan, LD Faddeev, MG Gasimov, YM Berezan and others. They were considered by the theorem on the existence of capacity in the inverse problem of spectral analysis for ordinary differential equations, and the formulation of the inverse spectral problem was to find a building for a given spectral function $\rho_{\alpha}(\lambda)$

Further A.I. Nachman, J. Sylvester, G. Uhlmann [7] published an article devoted to the proof of the theorem of multidimensional Borg - Levinson. Consider the following problem:

\author{
Kushkumbaeva A.S. \\ Nosov Magnitogorsk State Technical University, \\ the city of Magnitogorsk, Chelyabinsk oblast, Russia \\ e-mail: aiman-kysh@mail.ru
}

$$
\left\{\begin{array}{l}
(-\Delta+q) u=\lambda u \quad \text { в } \Omega, \\
\left.u\right|_{S}=0
\end{array}\right.
$$

where $\Omega$ - in a bounded domain $R^{n}(n \geq 2)$ with boundary of class $S C^{\infty}$, they proved the uniqueness of the building restoration $\mathrm{q}$ if aware of all the eigenvalues and values pro-water normal to the boundary $S$ of the eigenfunctions of this problem.

The fact that the theorem on the uniqueness of the solution of inverse problems include the value, do not affect the uniqueness of the RECOVER-building, became clear after work H.Isozaki [6]. He proved a theorem on the uniqueness of the reduction potential in the problem (1) if there is no finite number of spectral data.

Let $\Omega$ - in a bounded domain $R^{N}$, where $\mathrm{N} \geq 2$, with boundary of class $\mathrm{S} C^{\infty}$. Consider the Dirichlet boundary value problem with the actual function $q \in C^{\infty}(\bar{\Omega})$ with a spectral parameter $\lambda$ :

$$
\left\{\begin{array}{l}
-(\Delta u)(x)+q(x) u(x)=\lambda u(x), x \in \Omega, \\
u(x) /_{S}=0 .
\end{array}\right.
$$

A solution of problem (1) is a function of $C^{\infty}(\bar{\Omega})$, satisfying (1) everywhere on $\bar{\Omega}$. The solution to this problem will be considered in the space $L_{2}(\Omega)$.

It is known that the problem (1) has no more than a countable number of Own-governmental values, each of which actually and has finite multiplicity.

Let the eigenvalues $\mu_{1}, \mu_{2}, \ldots$ of (1) are numbered based on the increase and $m_{t}=m_{t}(q)$ - multiplicity of an eigenvalue $\mu_{t}(q)$. Across $u_{j t}, 1 \leq j \leq m_{t}$ denote the 
orthonormal eigenfunctions corresponding $\mu_{t}$. These feature sets are not uniquely defined. We believe

$$
E_{t}(q)=\left\{\left.\left(\frac{\partial \boldsymbol{u}_{1 t}}{\partial v}(q), \frac{\partial \boldsymbol{u}_{2 t}}{\partial v}(q), \ldots, \frac{\partial \boldsymbol{u}_{m_{t} t}}{\partial v}(q)\right)\right|_{S}\right\}
$$

where $v$ - the outer normal to the surface $\mathrm{S}$. We consider two systems of eigenfunctions

$$
\left\{u_{1 t}, u_{2 t}, \ldots, u_{m_{i} t}\right\},\left\{v_{1 t}, v_{2 t}, \ldots, v_{m_{i} t}\right\}
$$

equivalent if there exists an orthogonal matrix $\mathrm{T}$, for which

$$
\left.\left(\frac{\partial u_{1 t}}{\partial v}, \cdots, \frac{\partial u_{m_{i} t}}{\partial v}\right)\right|_{S}=\left.\left(\frac{\partial v_{1 t}}{\partial v}, \cdots, \frac{\partial v_{m_{i} t}}{\partial v}\right)\right|_{S} \cdot T
$$

These equivalence classes denoted by $W_{t}$. Let the function $V \in C^{\infty}(\bar{\Omega})$, considered as an element of $L_{2}(\bar{\Omega})$ , it is the solution of the Dirichlet problem

$$
\left\{\begin{array}{l}
-(\Delta V)(x)+q(x) V(x)=\lambda V(x), x \in \Omega, \\
V(x) /_{S}=f,
\end{array}\right.
$$

where $f \in C^{\infty}(\bar{\Omega})$ considered as an element of $L_{2}(\Omega)$, and $\lambda \neq \mu_{t}(q), t=\overline{1, \infty}$. We introduce the operator Neumann $N: L_{2}(\Omega) \rightarrow L_{2}(S)$ using equality

$$
N(\lambda, q) f=\left.\frac{\partial V}{\partial v}\right|_{S}
$$

Consider the problem (1) the potentials $q_{1}, q_{2} \in C^{\infty}(\bar{\Omega})$. We define the scattering function

$$
F\left(\lambda, \omega, \theta ; q_{j}\right)=\int_{S}\left(N\left(\lambda, q_{j}\right) \varphi_{\lambda, \omega}\right)(x) \overline{\varphi_{\lambda,-\theta}(x)} d S_{x},
$$

where $S_{x}$ surface area of the element, $\varphi_{\lambda, \omega}(x)=\exp (i \sqrt{\lambda} \omega \cdot x), \quad \lambda \in C /(-\infty ; 0)$, $\omega, \theta \in S^{N-1}, \omega \cdot x=\sum_{r=1}^{N} \omega_{k} x_{k}, j=1,2$. It is known that

$$
\begin{gathered}
F\left(\lambda, \omega, \theta ; q_{j}\right)= \\
=-\frac{\lambda}{2}(\theta-\omega)^{2} \int_{\Omega} \exp (-i \sqrt{\lambda}(\theta-\omega) x) d x+\int_{\Omega} \exp (-i \sqrt{\lambda}(\theta-\omega) x) q_{j}(x) \\
=\int_{S}\left(-\Delta+q_{j}-\lambda\right)^{-1}\left(q_{j} \varphi_{\lambda, \omega}\right)(x) \cdot \overline{\varphi_{\lambda,-\theta}(x)} d S_{x} .
\end{gathered}
$$

Let $\xi \in R^{N}, \quad \xi \neq 0$ - an arbitrary fixed vector. Choose a $\eta \in R^{N},|\eta|=1$, so that $\eta$ orthogonal $\xi$.

We define for sufficiently large natural setting $n$ the following sequences:

$$
c_{n}=\left(1-\frac{|\xi|^{2}}{4 n^{2}}\right)^{\frac{1}{2}}, \theta_{n}=c_{n} \eta+\frac{\xi}{2 n}, \omega_{n}=c_{n} \eta-\frac{\xi}{2 n}
$$

$l_{n}=(n+i)^{2}$.

Here and everywhere below i - imaginary unit.

With this choice of variables $l_{n}, \theta_{n}, \omega_{n}, c_{n}$ have the equality [3]:

$$
\begin{aligned}
& \lim _{n \rightarrow \infty} F\left(l_{n}, \theta_{n}, \omega_{n} ; q_{j}\right)=-\frac{|\xi|^{2}}{2} \int_{\Omega} \exp (-i x \cdot \xi) d x+ \\
& +\int_{\Omega} \exp (-i x \cdot \xi) q_{j}(x) d x
\end{aligned}
$$

Some degree Neumann operator is an integral operator. Neumann kernel of formally given by the equation:

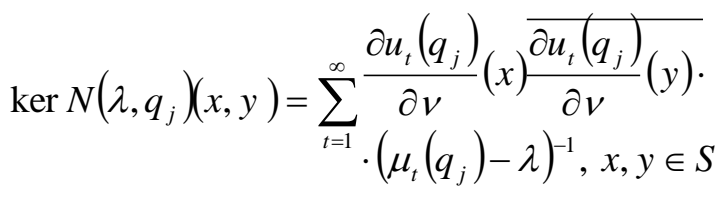

Difference $\operatorname{ker} N\left(\lambda, q_{1}\right)-\operatorname{ker} N\left(\lambda, q_{2}\right)$ it is the kernel of an operator in $L_{2}(S \times S)$. Then the definition of the scattering function,

$$
\begin{gathered}
\left|F\left(\lambda, \theta, \omega ; q_{1}\right)-F\left(\lambda, \theta, \omega ; q_{2}\right)\right| \leq \\
\leq\left\|N\left(\lambda, q_{1}\right)-N\left(\lambda, q_{2}\right)\right\| \cdot\left\|\varphi_{\lambda, \omega}\right\|_{L_{2}(s)} \cdot\left\|\varphi_{\lambda,-\theta}\right\|_{L_{2}(s)} \cdot
\end{gathered}
$$

Showing that

$\left\|\operatorname{ker} N\left(\lambda, q_{1}\right)-\operatorname{ker} N\left(\lambda, q_{2}\right)\right\| \rightarrow 0$ at $n \rightarrow \infty$

we can say, using equation (2) that $\lim _{n \rightarrow \infty}\left|F\left(l_{n}, \theta_{n}, \omega_{n} ; q_{1}\right)-F\left(l_{n}, \theta_{n}, \omega_{n} ; q_{2}\right)\right|=0$, and hence $q_{1}(x)=q_{2}(x)$ for anyone $x \in \bar{\Omega}$. Let the dimension of the space is equal to 2 or 3 . For sufficiently large natural $\mathrm{T}$ at

$W_{t}\left(q_{1}\right)=W_{t}\left(q_{2}\right)$

The requirement (3) is executed if the condition is true: 


$$
\begin{aligned}
& \lim _{n \rightarrow \infty}\left\|\sum_{t=T+1}^{\infty} \frac{\frac{\partial u_{t}\left(q_{1}\right)}{\partial v}(x) \frac{\overline{\partial u_{t}\left(q_{1}\right)}}{\partial v}(y)\left(\mu_{t}\left(q_{2}\right)-\mu_{t}\left(q_{1}\right)\right)}{\left(\mu_{t}\left(q_{1}\right)-l_{n}\right)\left(\mu_{t}\left(q_{2}\right)-l_{n}\right)}\right\|_{L_{2}(S \times S)}= \\
& =0 .
\end{aligned}
$$

Academician VA Ilyin and IA Shishmarev in [2] it was shown that

$$
\left\|\frac{\partial u_{t}}{\partial v}\left(q_{j}\right)\right\|_{L_{2}(S)}=O\left(\left[\mu_{t}\left(q_{j}\right)\right]^{N / 4}\right), j=1,2 .
$$

By Theorem Hormander we have: $\mu_{t} \sim C_{3} \cdot t^{\frac{2}{N}}$ at $t \rightarrow \infty$,

Therefore, we can say that

$$
\left\|\frac{\partial u_{t}}{\partial v}\left(q_{j}\right)\right\|_{L_{2}(S)}=O\left(t^{1 / 2}\right), \quad j=1,2
$$

Thus, the requirement (4) is valid if

$$
\lim _{n \rightarrow \infty} \sum_{k=1}^{\infty} \frac{t_{k}}{\left|\mu_{t_{k}}\left(q_{1}\right)-l_{n}\right|\left|\mu_{t_{k}}\left(q_{2}\right)-l_{n}\right|}=0 .
$$

Let subsequence of eigenvalues of the problem (1) satisfy the condition

$$
\forall k \in N\left(\frac{t_{k+1}}{t_{k}} \geq \lambda>1\right)
$$

What sequence called lacunar. In addition, we consider arbitrary strictly increasing sequence of positive integers, satisfying the condition

$$
\lim _{k \rightarrow \infty} \frac{t_{k+1}-t_{k}}{\sqrt{t_{k}}}=+\infty
$$

It can be shown that with this choice of the sequence, the requirement (4). Theorem.

Theorem 1. Let $\Omega$ - in a bounded domain $R^{2}$, with boundary $\mathrm{S}$ class $C^{\infty},\left\{\mu_{t_{k}}\left(q_{j}\right)\right\}_{k=1}^{\infty}, j=1,2$, subsequence of eigenvalues of the problems (1) the potentials $q_{j}$, the properties (5) or (6). If $q_{1}$ and $q_{2}$ such that for their own orthonormal functions Dirichlet problems (1) the following conditions are met with appropriate potentials:

There is a sufficiently large natural $\mathrm{T}$ such that when $t \geq T$ have the equality $W_{t}\left(q_{1}\right)=W_{t}\left(q_{2}\right)$.

$$
\mu_{t}\left(q_{1}\right)=\mu_{t}\left(q_{2}\right)_{\text {at }} t \neq t_{k},
$$

for any x of $\bar{\Omega}$ have the equality $q_{1}(x)=q_{2}(x)$.

Consider now the theorem on the uniqueness of the potential recovery in the event.

The same is true for the statement of the theorem in this case.

Theorem 2. Let $\Omega$ - in a bounded domain $R^{N}, N=3$, with boundary $\mathrm{S}$ class $C^{\infty},\left\{\mu_{t_{k}}\left(q_{j}\right)\right\}_{k=1}^{\infty}, j=1,2$, infinite subsequence of eigenvalues of the problems (1) the potentials $q_{j}$, indices which satisfy the requirements of:

$t_{k+1}>t_{k}+a_{k} t_{k}^{\frac{5}{6}}, \lim _{k \rightarrow \infty} a_{k}=+\infty, \sum a_{k}^{-\frac{1}{3}}<+\infty$.

If $q_{1}$ and $q_{2}$ such that for their own orthonormal functions Dirichlet problems (1) the following conditions are met with appropriate potentials:

1. there is a sufficiently large natural $\mathrm{T}$ such that when $t \geq T$ have the equality $W_{t}\left(q_{1}\right)=W_{t}\left(q_{2}\right)$.

2. $\mu_{t}\left(q_{1}\right)=\mu_{t}\left(q_{2}\right)$ at $t \neq t_{k}$, then for any $\mathrm{x}$ in $\bar{\Omega}$ have the equality $q_{1}(x)=q_{2}(x)$.

Conclusion.

1. We prove that there are infinite sequence of eigenvalues, do not affect the uniqueness of liability reduction potential in the problem of the Borg - Levins-on with Dirichlet boundary conditions. Infinite sequence-of, do not affect the uniqueness of recovery poten-tial for boundary value problems of the 2 nd and 3 rd type were obtained in [1], [3]- [9].

2. Condition (5) satisfy the sequence $t_{k}=\left[a \cdot k^{b}\right]$ at $a>0, b>2$. Condition (7) satisfy the same type of sequence, but $b>6$. And both conditions are satisfied by the sequence $t_{k}=\left[c \cdot d^{k}\right]$, where $c>0, d>1$, which is a lacunar.

\section{References}

[1]. VV Dubrovsky A theorem on the uniqueness of the solution of inverse problems of spectral analysis / VV. Dubrovsky-// differentials equations. - 1997. - Vol. 33, number 3. - S. 421 - 422.

[2]. VA Ilyin, IA Shishmarev Uniform in the closed domain of the eigenfunctions of an elliptic operator and their derivatives // Math. AN SSSR, Ser. Mat. - 1960. T. 24. S. 883 - 896.

[3]. Smirnov LV On the question of a mathematical model of the renewal of the smooth potentials in the back of the Dirichlet problem for the ndimensional case / LV Smirnova // Mathematical and software systems in the industrial and social spheres. - 2013. - № 1 (3). - S. 11-17.

[4]. Torshina OA The traces of discrete operators partial differential-water / OA Torshin // Almanac of modern science and drawing-education. Scientific theory / magazine theme. - 2012.- № 4 (59). - S. 220-222. 
[5]. Torshina OA The formula of the regularized trace for the Laplace first opera torus - Beltrami operator with potential on the non-smooth projective plane / OA Torshin // Vestnik of Samara State Technical University. Mathematics. - 2006. - S.32-40.

[6]. Isozaki H. Some remarks on the multidimensional Borg - Levinson theorem / H. Isozaki // J. Math. Kyoto Univ. (JMK YAZ). - 1991. V.31, № 3. - P. 743 - 753 .

[7]. Nachman, F.An n - Dimensional Borg - Levinson Theorem / F. Nachman, J. Sylvester, G. Uhlmann // J. Math. Phys. - 1988. - V. 115. P. 595 - 605.

[8]. Sadovnichii, V.A. Uniqueness of solutions to inverse eigenvalue problems / V.A. Sadovnichii, V.V. Dubrovskii, L.V. Smirnova // Doklady Mathematics. 2000.T. 61. № 1. C. 67-69.

[9]. Smirnova, L.V.Infinite sequences not affecting the unique recovery of the potential / L.V.Smirnova, O.A.Torshina // Applied and Fundamental Studies: Proceedings of the 4th International Academic Conference. November 29-30, 2013, St. Louis, USA. Publishing House Science and Innovation Center, Ltd., -2013. 